\title{
La migración internacional y los cambios en las relaciones de género y estructuras de los hogares: la migración colombiana hacia España
}

\author{
Jeanny Posso Quiceno ${ }^{1}$ \\ Fernando Urrea Giraldo ${ }^{2}$ \\ Universidad del Valle. Departamento de Ciencias Sociales
}

\section{Resumen}

El artículo analiza el funcionamiento de las redes de hogares transnacionales de la migración colombiana hacia España y los cambios en las relaciones de género en diferentes dimensiones de la vida de mujeres y hombres migrantes. El artículo enfatiza las diferencias sociales entre los migrantes hacia España y los Estados Unidos, al igual que los miembros de los hogares que residen en Colombia. Mientras los primeros - tanto los migrantes como sus familiares en Colombia - presentan un considerable nivel educativo menor, los segundos tienen un nivel mayor. Esto, a la vez, se corresponde con las áreas geográficas de salida desde las regiones del Valle del Cauca y Cauca, que tienen la mayor participación porcentual en la inmigración hacia España. Así, el peso principal de la inmigración hacia este país está constituido por clases populares y clases medias empobrecidas de una región con alta concentración de población negra, sin que ellas correspondan a los segmentos más pobres de la población colombiana.

La parte central del artículo describe las transformaciones de género, del campo afectivo y de la vida sexual, particularmente en el caso de las mujeres, que estos hogares transnacionales vehiculan, en relación con el uso de los recursos monetarios de que disponen los hogares, gracias a la participación femenina en el mercado de trabajo, a pesar de que tienen empleos no calificados y muy precarios, la mayor parte de ellos relacionados con el servicio doméstico en la sociedad española. El artículo revela las tensiones entre las mujeres inmigrantes y sus parejas en la decisión de emigrar y en el tránsito hacia la reagrupación familiar, al igual que los conflictos con el entorno familiar que dejan en Colombia los migrantes.

Palabras clave: redes de hogares transnacionales, relaciones de género, clases populares urbanas, campo afectivo y vida sexual.

1. Antropóloga, profesora asistente, Departamento de Ciencias Sociales, Facultad de Ciencias Sociales y Económicas, Universidad del Valle. En la actualidad, lleva a cabo el proyecto de investigación Redes familiares transnacionales, los reacomodos en los ámbitos productivo y doméstico entre hombres y mujeres en las familias de emigrantes colombianos a España. La información cualitativa de este artículo se apoya en las entrevistas realizadas por esta investigadora.

2. Sociólogo, profesor titular, Departamento de Ciencias Sociales, Facultad de Ciencias Sociales y Económicas, Universidad del Valle.

Se contó con la colaboración del estudiante de Sociología de la Universidad del Valle, Jairo Alexander Castaño López. 
Abstract. International migration and changes in gender relations and household structures: Colombian migration to Spain

This article analyzes the nets of transnational households of the Colombian migration toward Spain and the changes into the gender relationships in different life dimensions of migrants, women and men. The article emphasizes the social differences among the migrants toward Spain and the United States, the same as the members of their households that reside in Colombia. While the first ones — as much the migrants as their relatives in Colombia - present a considerable smaller educational level, the seconds have a bigger level. This at the same time belongs together with the geographical areas, the Valle of Cauca and Cauca region, which they have the bigger percentage in the immigration toward Spain. So, the main weight of the Colombian immigration in this country is constituted by popular classes and impoverished middle classes of a region with high concentration of black population, without they correspond to the Colombian population's poorer segments.

The central part of the article describes the gender transformations, the affective and sexual life fields, particularly in the case of women that these households transnational carry, in connection with the use of monetary resources that they get, thanks to the women participation in the labor market, although they work in the lowest qualified employments and the most precarious, related with the domestic service activities in the Spanish society. The article reveals the tensions between the immigrant women and its couples in the decision of emigrating and also in the family regrouping process, the same as the conflicts with the family environment that they leave in Colombia.

Key words: nets of transnational households, gender relationships, urban popular classes, affective and sexual life fields.

\section{Sumario}

Introducción: el contexto de la migración colombiana hacia España

Algunos factores de la oleada reciente de emigración internacional hacia España Algunas características sociodemográficas y socioeconómicas de los migrantes en España y de los miembros de sus hogares en Colombia

Un acercamiento a partir de estudios de caso a la conformación de redes de hogares transnacionales en España
La división sexual del trabajo en el grupo doméstico transnacional: el antes y el después del proceso migratorio

Nuevos ingresos y las relaciones de género en la gestión de los recursos del hogar transnacional

La recomposición de los afectos y la sexualidad

Conclusiones

Bibliografía 
A pesar de que estaba sola en España, ya tenía como una vida nueva hecha. Yo me iba a trabajar, salía de trabajar y, si quería, me iba pa'l Internet o me iba a andar a la calle, me iba a recorrer las vitrinas. Que así estuvieran los niños lejos, pero vivía como en paz. (Diana Martínez, mujer blanca colombiana nacida en Cali, 38 años, trabajadora doméstica en $\mathrm{Madrid}^{3}$ )

\section{Introducción: el contexto de la migración colombiana hacia España}

De acuerdo con los resultados del censo de 2005 realizado en Colombia, a 31 de diciembre del año pasado, los colombianos que se encuentran en el exterior llegan a 3.331.107 de personas, de los cuales, por lo menos, alrededor del sesenta por ciento salieron de Colombia en los últimos ocho años. De ellos, el 23,4\% se encuentran en España, o sea, 779.479 personas. Este país es, hoy en día, el segundo en importancia, después de los Estados Unidos (con el $35,4 \%$ ), en recepción de emigrantes colombianos, desplazando a Venezuela al tercer lugar (con el 18,5\%). Por otro lado, según el estudio de Aparicio y Giménez (2003), el 29,3\% de los migrantes colombianos en España provienen del Departamento del Valle del Cauca, con lo que constituye la región colombiana de mayor concentración de salida hacia ese país, sobre todo desde finales de la década de 1990. En general, todos los municipios de esta región tienen hogares con migrantes en España, sobresaliendo la ciudad de Cali y los municipios que conforman su área metropolitana, como es el caso del municipio de Candelaria. Igualmente ocurre con los municipios de la región del norte del Cauca, que forman parte también del área metropolitana de Cali (Puerto Tejada, Santander de Quilichao, Caloto, entre otros). En estos casos, como puede observarse en los resultados del censo 2005, los hogares en los municipios del entorno metropolitano de Cali (que a la vez constituyen «ciudades dormitorio» respecto a Cali), hoy en día tienen más migrantes en España que en otros países, incluso respecto a los Estados Unidos.

En la emigración colombiana hacia España, como era de esperar, Garay y Rodríguez (2005a: 48) advierten que está constituida por redes de parientes, concentrada «[...] relativamente en los estratos bajos como resultado de la búsqueda en el exterior de mejores oportunidades laborales y de ingreso para sus hogares: emigrando el padre y/o la madre en edad de trabajar así sea a costa de su "separación temporal" y eventual reagrupamiento futuro de la familia en el país de destino. A diferencia, en el caso de los estratos altos con educación profesional y especializada, la emigración tiende a realizarse de una manera más ordenada y previsiva sin correr riesgos y sacrificios excesivos a nivel individual y familiar, gracias a sus mejores condiciones como familia para insertarse en la sociedad de destino de manera regular, formal y con mejores perspectivas laborales y de ingreso». En el caso de la migración colombiana hacia

3. Entrevista realizada en Cali en febrero de 2006, en su primer viaje de vacaciones después de cinco años. 


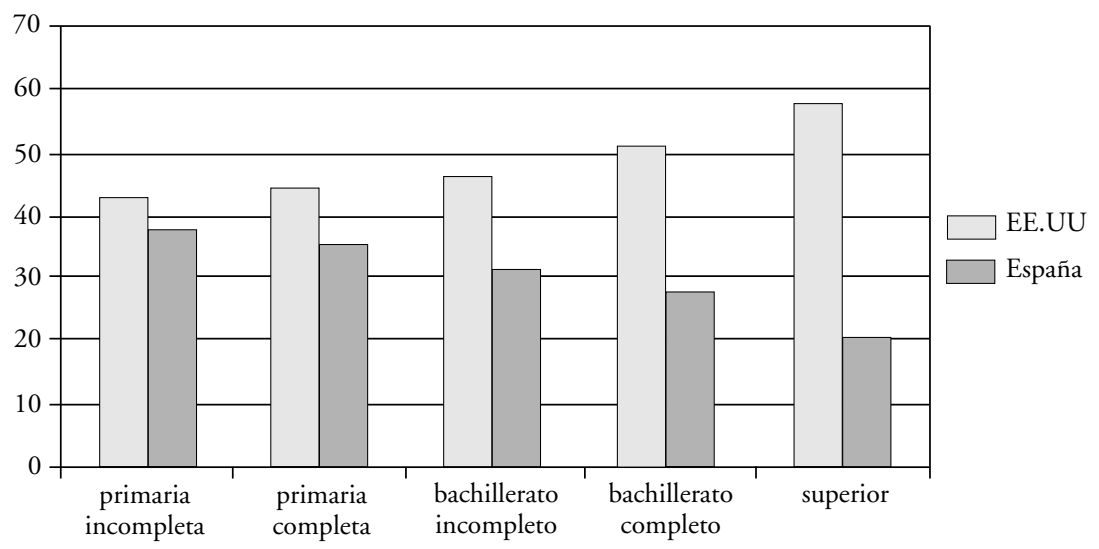

Gráfico 1. Remesas desde Estados Unidos y España, por nivel educativo de los receptores (porcentajes), 2004. Fuente: Garay y Rodríguez (2005a: 55).

España, desde las diferentes regiones del Valle del Cauca y del norte del Cauca, para la mayoría de los hogares, corresponde típicamente al primer grupo, sin desconocer la presencia de migrantes en hogares de estratos socioeconómicos en mejores condiciones. Los mismos autores aportan datos que indican que los receptores de las remesas para los departamentos de la región Pacífica (departamentos del Valle del Cauca, Cauca, Nariño y Chocó) decrecen a medida que aumenta el nivel educativo, es decir, que se concentran en los grupos de menor nivel educativo, al contrario de lo que ocurre en otras regiones del país, como Bogotá y la región del Atlántico (op. cit.: 53). También demuestran que la escolaridad de los migrantes colombianos hacia Estados Unidos es mayor que la de los migrantes hacia España (ver gráfico 1), para todas las regiones de Colombia, pero este fenómeno sería más acentuado en la región Pacífica.

\section{Algunos factores de la oleada reciente de emigración internacional hacia España}

¿Cuáles son los factores que explican la importante participación porcentual de emigrantes colombianos en España procedentes de departamentos como el Valle del Cauca y Cauca? La intensa crisis económica hacia finales de los años noventa en el país tuvo mayores repercusiones o intensidad en la región del Valle del Cauca y, en general, en toda la región Pacífica (Urrea y Ortiz, 1999). Por otro lado, la opción de trasladarse a un país en el que se habla la misma lengua, al tiempo que en éste se registra un continuo aumento de demanda de mano de obra no calificada y semicalificada, sobre todo en el sector de los servicios, la construcción, el sector turístico y de restaurantes, entre otros. Inicialmente, sin 
visado, luego, su expansión ante la presión migratoria facilitó que determinados sectores de población de clases medias bajas y bajas con menor capital escolar y menores recursos económicos de regiones como el Valle del Cauca en Colombia se orientaran hacia España, incluso después de la obligación del visado. De este modo, a medida que se afincaron los primeros migrantes en algunas ciudades y localidades españolas hacia finales de los años noventa, ellos constituyeron la plataforma de una serie de redes de acogida que siguieron conectando nuevos migrantes, fenómeno similar para las diferentes nacionalidades de migrantes. Tampoco se pueden dejar de lado otros factores coyunturales que han tenido que ver con ello, por ejemplo: el impacto de lo sucedido con las torres gemelas en Nueva York el 11 de septiembre y sus efectos sobre un mayor cierre de las políticas inmigratorias de Estados Unidos, que posiblemente reorientaron la emigración hacia Europa por parte de un segmento de la población potencialmente emigrante. Esto último es igualmente válido para emigrantes de otras nacionalidades latinoamericanas y demás continentes.

Los resultados preliminares del censo de 2005 indican que el Departamento del Valle del Cauca constituye la principal región colombiana con emigrantes internacionales, siendo mayoritaria en esta región la emigración hacia España ${ }^{4}$ y, en el caso de $\mathrm{Cali}^{5}$, pueden llegar al 30\% de los hogares de esta ciudad. Ahora bien, respecto a los hogares con miembros migrantes en España, se moverían entre el 3\% y el 5\% del total de los hogares colombianos, especialmente concentrados en algunas regiones de Colombia como el Valle del $\mathrm{Cauca}^{6}$, como se ha analizado previamente, lo cual revela un interesante impacto que esta migración tiene en algunas áreas de la geografía colombiana y en determinadas capas sociales de las clases populares de la población. Por lo mismo, si bien es probable que los hogares más acomodados cuenten con más miembros con experiencia migratoria internacional, mientras los hogares de sectores populares más pobres tienen una participación mucho menor en esta experiencia, como parecen mostrarlo los datos preliminares del censo de 2005, de todas maneras, llama la atención que en la dinámica de migración internacional se abre un espacio también para sectores de las clases populares.

Por supuesto, un análisis de factores internos desde el país de salida, al igual que la importancia o la magnitud del fenómeno de emigración internacional para los hogares en la sociedad de origen hacia un determinado país receptor (en nuestro caso, España), tiene que verse en el marco de los procesos de globalización del capitalismo contemporáneo.

4. Según datos del censo de 2005, el 24,14\% de los hogares del Valle del Cauca tienen migrantes en el exterior, de los cuales casi el $40 \%$ están en España.

5. Cali es la capital del departamento del Valle y una de las tres ciudades con mayor número de habitantes de Colombia, en el 2005 contaba con 2.068.386 habitantes (datos definitivos del censo de 2005 sin ajuste de cobertura).

6. Según nuestros cálculos, apoyados en los últimos resultados del censo colombiano, alrededor del 10\% de los hogares del Valle del Cauca y de la ciudad de Cali tienen migrantes en España. 


\section{Algunas características sociodemográficas y socioeconómicas de los migrantes en España y de los miembros de sus hogares en Colombia}

Como señalan los estudios realizados sobre los colombianos en España (Aparicio y Giménez, 2003; Garay y Rodríguez, 2005b), se trata de una migración más femenina que masculina (por encima del $54 \%$ ), fenómeno más pronunciado que en el caso de la emigración hacia los Estados Unidos, aunque también hacia este país más del $50 \%$ son migrantes mujeres. El estudio de Aparicio y Giménez (2003: 32) arroja que un poco más del 60\% se encuentra en la franja de edad de 25-44 años, seguida del grupo entre 19-24 años (13\%). Tendencia similar arrojan los datos del estudio de Baca y otros (2005). Por ser una migración más reciente, también el promedio de edad de los migrantes en España es casi cinco años menor al de los colombianos en Estados Unidos (34,33 años versus 39,55 años, datos Baca y otros, análisis en Garay y Rodríguez, 2005b). En este sentido, también hay una mayor participación significativa de solteros en los migrantes a España (Garay y Rodríguez, 2005b: 25) que a Estados Unidos, y lo más importante, los datos también revelan que España concentra una alta proporción de migrantes colombianos mujeres que han dejado a sus hijos o a su esposo o compañero en Colombia, y también hombres respecto a su esposa o compañera (el 11,7\% de los emigrantes de la encuesta Amco-DANE, ver Baca y otros, versus solamente el 5,9\% en el caso de Estados Unidos) (op. cit.: 25).

Esto último muestra claramente un fenómeno de un modelo migratorio de hogares transnacionales, con miembros del núcleo familiar primario entre los dos países, correspondiente a un ciclo de la migración internacional en sus fases tempranas, previo a iniciarse el ciclo posterior de reunificación familiar. Pero lo que hay que destacar es el peso porcentual de las mujeres bajo esta condición, mucho mayor que el de los hombres, al igual que en la categoría de soltero. En Estados Unidos, según los autores referenciados (op. cit.: 25), en cambio, hay una mayor participación de migrantes colombianos casados o en unión libre, hombres y mujeres, lo que se corresponde con la presencia de cohortes de migrantes que llevan más de diez o quince años residiendo en ese país.

En términos sociolaborales, de ciclo de vida y de posición social, de las entrevistas realizadas en hogares con migrantes en España, en Cali y en algunas de las "ciudades dormitorio" del área metropolitana (Candelaria y Puerto Tejada), los hombres migrantes eran en Colombia, antes de viajar, operarios calificados y no calificados en el sector industrial (grandes y medianas empresas), tanto empleados como desempleados, entre 25 y 45 años. Los hombres y las mujeres más jóvenes (entre 18 y 25 años) eran bachilleres, técnicos o tecnólogos recién egresados o en empleos ocasionales e informales. En el caso de las mujeres entre 25 y 45 años, se presenta un amplio grupo de ocupadas en empleos del sector público, dueñas de pequeños negocios, secretarias y en cargos administrativos medios y bajos del sector privado, pero también trabajadoras de la confección. 


\section{Un acercamiento a partir de estudios de caso a la conformación de redes de hogares transnacionales en España}

Los ejemplos que se presentan a continuación en este trabajo, ejemplifican muy bien la incorporación masiva de capas de sectores populares en la migración colombiana a España. Se trata de seis casos, mujeres y hombres de sectores de clase media baja del área metropolitana de Cali y del municipio de Cartago, que no llevan más de seis años en España. Son los siguientes:

- El hogar de los Castillo: conformado por una madre sola (44 años) y sus tres hijos. Al momento de emigrar (la madre) en el 2000, tenían 18 y 17 años las dos hijas y 13 el menor. Viven en un barrio de clases populares en las afueras de Palmira ${ }^{7}$. Martha, la madre, es una mujer mestiza ${ }^{8}$, con educación secundaria incompleta. Años atrás, había sido obrera del sector de la confección en un distrito industrial de producción de maquila.

- Los Bejarano: constituidos por una pareja y sus dos hijos en un barrio de la ciudad de Cali. Diana, la madre, inicia el proceso migratorio en el año 2000. Ella es una mujer blanca que inicialmente se va hacia Costa Rica como refugiada política a raíz del asesinato de su padre. Desde este país, se traslada a España, con lo que pierde su estatus de refugiada. Diana había realizado estudios en el SENA ${ }^{9}$, en el área administrativa, y se había desempeñado como empleada en una entidad bancaria estatal durante más de diez años, de la que llegó a ser gerente de una sucursal, hasta el momento en que la entidad es adquirida por un banco español y se producen reajustes de plantilla que la dejan sin trabajo en el año 1999, lo que coincide con la aguda crisis económica de la región.

- Los Caicedo: formados por una pareja y sus dos hijos de un barrio de la ciudad de Cali. El padre viaja inicialmente en el año 2000. Iván es un hombre mulato, tornero industrial formado en el SENA, que, años atrás, había sido emigrante en Venezuela y Ecuador. La madre, Liliana, una mujer mestiza con educación primaria y obrera del sector de la confección, viaja siete meses después cuando recibe la noticia de que su marido había sufrido un gravísimo accidente laboral que lo tenía entre la vida y la muerte. Al momento de viajar, Iván tiene 44 años y su esposa 45 , los niños ${ }^{10}$, dos varones, tienen 15 y 6 años.

- El hogar de los Fuentes: se trata de una pareja, él blanco y ella mestiza, y su hijo. Antes vivían en un barrio de la ciudad de Cali. Emigra el padre, César,

7. Palmira es una ciudad intermedia que forma parte del área metropolitana de Cali, situada al límite norte de ésta, que contaba en el 2005 con 278.409 habitantes (datos del censo de 2005 sin ajuste de cobertura).

8. Los tres hijos de Martha son mulatos.

9. Servicio Nacional de Aprendizaje, entidad pública del nivel nacional que brinda formación profesional y, guardadas las proporciones, realiza algunas funciones similares al INEM en España.

10. Los hijos de Iván y Liliana son mestizos. 
en el año 2001 con 35 años, su esposa, Mariela en ese momento tenía 29 años y su hijo, 5. César tiene estudios secundarios, en los últimos años antes de emigrar, vivía de empleos eventuales, también trabajó como taxista. Mariela tiene algunos años de universidad, aunque no terminó su licenciatura en Economía. Ella es una comerciante que tiene su propio negocio de alquiler de vídeos con el que se garantizan ingresos estables para la familia. En el momento, César es cocinero en Tenerife y Mariela, desde Cali, adelanta las gestiones para hacer la reagrupación familiar junto con su hijo.

- El hogar de tres mujeres de apellido Arias: Olga, una mujer mestiza de 26 años, vivía con sus dos hijas ${ }^{11}$, de 3 y 10 años en el momento de viajar en el año 2002, en un barrio de clase media baja del municipio de Cartago ${ }^{12}$ (Valle); con bachillerato incompleto, trabajaba como secretaria para una entidad pública ganando el salario mínimo. El padre de sus hijas, después de años de ausencias y retornos, había iniciado el año anterior convivencia con otra pareja. Olga, hoy en día, trabaja haciendo limpieza por horas y está realizando los trámites para reagrupar a sus hijas. También tiene proyectos de matrimonio con un hombre peruano con el que convive en Aluche (Madrid).

- Los Murillo ${ }^{13}$ : Andrés un habitante de Candelaria ${ }^{14}$ (Valle) es un hombre mestizo, que durante el 2000, cuando aún está soltero con 24 años, viaja hacia Aruba. Las dificultades por no hablar inglés lo llevan a cambiar su destino migratorio hacia España, a donde arriba en el 2001. Allí se dirige hacia Marbella, donde se encuentra una amiga de infancia que lo aloja los primeros días, el esposo de ella (también de Candelaria) le ayuda a conseguir trabajo. Por medio de ellos, conoce a Paula, colombiana de 22 años y originaria también de Candelaria, con la que, cinco meses después, conformará una pareja de hecho. Dos años más tarde, tienen una hija y en la actualidad continúan viviendo todos en Marbella.

\section{La división sexual del trabajo en el grupo doméstico transnacional: el antes y el después del proceso migratorio}

Los casos encontrados y analizados muestran una situación en la que predomina la incapacidad de los varones para cumplir con el rol tradicional de proveedor económico, lo que se ha descrito como «la erosión del papel del varón

11. Las hijas de Olga también son mestizas.

12. Cartago es un municipio localizado al norte del departamento del Valle que para el 2005 arroja 139.450 habitantes (proyecciones de población del DANE en espera de los datos definitivos censales del 2005).

13. Información recogida por el estudiante de sociología Jairo Alexander Castaño.

14. Candelaria es un municipio que hace parte del área metropolitana de Cali, situada al límite oriental de la ciudad, que para el 2005 tenía 69.008 habitantes (proyecciones de población del DANE en espera de los datos definitivos censales del 2005). 
como proveedor económico» (Ramírez y otros, 2005: 7). Las mujeres, que en Colombia ya participaban en el mercado de trabajo como obreras, empleadas del sector servicios, microempresarias, o en el trabajo informal realizado en el domicilio, han estado cubriendo una parte o la mayor parte de los requerimientos económicos del hogar. Esta situación empuja a migrar tanto a hombres como a mujeres, porque ellas, aunque lleguen a tener algunas veces una mayor estabilidad en el empleo o en las actividades económicas que desarrollen, no alcanzan a cubrir todos las necesidades familiares con sus ingresos, especialmente si hay más de un hijo, lo que lleva a que, en una gran parte de los casos, sean ellas las que hayan tomado la iniciativa de emigrar.

En cuatro de los casos, los hombres habían adoptado una estrategia de huida. Por ejemplo, en el hogar de los Castillo, trece años atrás, el marido había emigrado a Estados Unidos, desentendiéndose paulatinamente del sostenimiento económico de sus hijos y, posteriormente, iniciando una nueva relación de pareja. Olga, cabeza de la familia Arias, durante años, fue la sufragadora principal de los gastos de la familia, su marido estaba ausente la mayor parte del tiempo y pocas veces hacia aportes económicos. Un año antes de su viaje, Olga se enteró de que su esposo acababa de tener un hijo con otra mujer y, al poco tiempo, él se fue a vivir con su nueva pareja. Esta situación empujó a estas mujeres cabeza de familia a buscar una forma de obtener un mayor ingreso del que podrían obtener en Colombia. También, en los hogares de los Bejarano y los Caicedo, los hombres asumieron una actitud de evasión que se expresaba en el consumo de licor y la infidelidad, mientras tanto sus esposas asumían la mayor parte de la carga económica del mantenimiento de la familia, lo que, como veremos más adelante con detalle, fue un ingrediente más en la decisión de emigrar de la mujer en el primer caso y del marido en el segundo.

Desde el punto de vista de la división sexual del trabajo remunerado, encontramos que, en los casos en que se trata de parejas, la migración ha permitido a los hombres recuperar su rol como proveedores principales, pues en la sociedad de acogida, en los tres casos analizados, los empleos masculinos que encuentran los inmigrantes están mejor remunerados que los femeninos. Esto ha implicado que, aunque en las fases iniciales del proceso migratorio, las mujeres han trabajado en jornadas intensas y agotadoras para compensar esos salarios inferiores, cuando los hombres se incorporan al mercado de trabajo, ellas reducen su jornada, retornando a un rol más tradicional. Estas mujeres consideran la nueva situación como un relevo en la responsabilidad como proveedoras principales que les llega a representar un alivio, una mejor situación frente a la doble jornada que siempre han desempeñado, pues, aunque siempre han trabajado, han sido las responsables del rol doméstico en el hogar. En este sentido, una reducción en las horas laboradas en el mercado de trabajo representa una mejor calidad de vida para ellas. Veamos dos de los hogares en los que se presenta esta situación.

Un ejemplo es el de la familia Bejarano. En Colombia, la esposa era la principal proveedora económica del hogar, ella emigra cuando pierde su trabajo en una entidad bancaria. Diana inicialmente le propone al marido que 
emprenda el proyecto migratorio, pero éste se niega argumentando las razones del afecto hacia su esposa, por lo que ella emprende la aventura migratoria. Desde finales del primer año de su partida hasta el tercer año, Diana retoma el rol como proveedora principal del hogar en Colombia, con el compromiso de que su marido aportaría un tercio del presupuesto. Esta forma de funcionamiento entra en crisis porque ella percibe cada vez más que él no se esfuerza y aporta cada vez menos al presupuesto familiar. Diana había tenido que extender su jornada de trabajo hasta límites insostenibles ${ }^{15}$ para poder hacer frente a los gastos de su familia en Colombia, hasta que cae enferma por el exceso de trabajo. Antes de que la ruptura sea total, ella logra conseguir un precontrato para que Humberto pueda viajar a España con permiso de trabajo. Al llegar, él rápidamente logra conseguir un empleo con contrato de trabajo y mejor remunerado que el de Diana. Esto permite a Diana reducir su jornada de trabajo y a Humberto hacerse cargo de los gastos de la familia como proveedor principal.

En el caso de los Caicedo, encontramos un patrón parecido. En esta pareja, la inestabilidad laboral de él estaba afectando a la relación. De hecho, la decisión de Iván de emigrar hacia España estaba mediada por un ultimátum de su esposa, que lo apremiaba porque estaban a punto de perder la propiedad de la vivienda, debido a la incapacidad de Iván de hacer frente a las cuotas del banco (que eran su responsabilidad). Cuando él viaja, ella sigue a cargo de la responsabilidad económica de la familia, pero en situación deficitaria, pues sus ingresos como obrera en el sector de la confección no alcanzaban a cubrirlo todo. Como se señaló antes, Liliana, al poco tiempo, viaja a España a causa de un accidente laboral de Iván, ella empieza a trabajar como empleada interna ${ }^{16} \sin$ papeles y de nuevo se hace cargo de los gastos del hogar ${ }^{17}$. Aproximadamente un año después, cuando Iván se restablece y puede incorporarse al mundo laboral, ella renuncia a su trabajo como interna y se hace cargo de la casa y de los hijos, que han logrado reunir con ellos. Poco tiempo después, ella realiza algunos trabajos eventuales, como la limpieza de escaleras o la hostelería, hasta que encuentra otro trabajo estable en el servicio doméstico como empleada externa ${ }^{18}$ y ya regularizada. En este caso, aunque Iván no había podido regularizarse y trabaja sin permiso de trabajo, logra encontrar una ocupación mejor remunerada que la de ella ${ }^{19}$.

15. Durante ese primer año y medio, ella trabaja atendiendo una línea de teléfono erótico en España, de 11 de la noche a 6 de la mañana, luego, en la mañana, hacía unas horas de limpieza y dormía un poco. En la tarde, de nuevo trabajaba en un turno del teléfono desde las 3 de la tarde hasta las 9 de la noche, luego volvía a dormir, con frecuencia seguía sin dormir hasta el otro día. En esa época, en promedio, sus horas de sueño apenas llegaban a tres diarias, ritmo que seguía todos los días incluidos domingos y festivos.

16. Viviendo en la residencia del empleador.

17. Tanto de los niños que están en Colombia, como de su esposo en Madrid.

18. Viviendo en su propio domicilio y desplazándose diariamente al lugar de trabajo en una jornada de seis horas al día.

19. Mientras ella gana seiscientos euros mensuales, él gana novecientos euros. 
En lo que concierne a la familia Fuentes, que corresponde a una pareja más joven, podría afirmarse que no tienen tan asumidos los roles tradicionales, pues es importante señalar que César no muestra una actitud de evasión respecto a sus responsabilidades. La pareja, al ser entrevistada, al unísono manifiesta el hecho de que ella es una mujer independiente económicamente y que ha sabido valerse perfectamente en la primera etapa de la migración de César. Al momento del viaje, su relación no se encontraba en crisis y se han mantenido unidos a pesar de los cinco años de ausencia de él. Esta pareja afronta la responsabilidad económica del hogar como un proyecto común.

La pareja más joven, Paula y Andrés, que ahora cuentan respectivamente con 27 y 30 años, se diferencia de los ejemplos anteriores porque sus miembros iniciaron la convivencia en el nuevo país de residencia. Los cambios que se han dado en este hogar en relación con el empleo de Paula, han dependido, por un lado, del hecho que Andrés ha logrado mejorar su salario y sus condiciones de trabajo después de asociarse con otros colombianos para hacer pequeños trabajos de reforma en el sector de la construcción. Este incremento en los ingresos del hogar permitió a Paula inicialmente cambiar su empleo en un restaurante por un trabajo a tiempo parcial, cuidando a personas mayores, y después interrumpir su vinculación durante un tiempo cuando se produjo el nacimiento del primer hijo. Sin embargo, esto no ha significado, en el terreno de lo doméstico, que Paula se haya hecho cargo de todas las tareas, la obligación para con el bebé es mutua y los cuidados deben de ser compartidos. Así mismo, ellos comparten las actividades de la organización de los espacios internos de la casa, de la dieta alimenticia y de las actividades familiares. En esta pareja, se nota desde su conformación un mayor equilibrio en las relaciones de poder, lo cual, de acuerdo con lo relatado por Paula, tiene mucho que ver con la primera etapa de su estancia en España, cuando vivía sola y aprendió a ser autosuficiente.

Por último, en el caso de las dos mujeres cabeza de familia, lo que se puede observar es la intensificación de la jornada laboral de ellas y el mayor esfuerzo comparado con las mujeres que están acompañadas para poder cubrir los gastos, tanto de las necesidades básicas del hogar como para cubrir el costo de las funciones reproductivas que, especialmente en el caso de la familia Arias, debe ser pagado. Un aspecto que es importante resaltar es que para las mujeres solas es mucho más difícil hacer la reagrupación familiar, por lo que el envío de remesas se extiende más en el tiempo frente a las parejas con hijos ${ }^{20}$.

20. Esta observación también pudo ser confirmada respecto a dos de las amigas más cercanas de los Bejarano, en uno de los casos, la mujer, que trabaja para una línea de telefono erótica, no ha podido llevar a su hijo de siete años, pues los requisitos para la reagrupación implican demostrar unos ingresos mínimos. En el otro caso, la compañera de piso de esta pareja viajó con ellos para dejar a su hija recién nacida al cuidado de la abuela materna. 


\section{Nuevos ingresos y las relaciones de género en la gestión de los recursos del hogar transnacional}

En este aspecto, habría que considerar dos períodos, especialmente en aquellos hogares en los que la pareja se ha logrado reunir en España, es decir, antes y después de la reagrupación, pero también dos cuestiones: la gestión del dinero en España y de las remesas. En este sentido, podríamos presentar los casos analizados en dos grupos, de un lado las parejas y, de otro, las dos mujeres solas.

En el primer grupo, podemos situar al hogar de los Bejarano. Diana, mientras su marido estaba en Colombia, gestionaba todo a través de su hermana y su madre. Ella enviaba el dinero a su hermana con las instrucciones precisas para que ella lo repartiera entre el pago de la deuda pendiente por su pasaje, el mantenimiento de los niños, su marido y su madre, el ahorro para comprar una casa y algunos gastos extras.

Yo no se lo mandaba a él ¿por qué? Por lo que yo había pasado anteriormente. Entonces yo decía, si se lo mando a él, lo va a despilfarrar en otras cosas y vamos a seguir con las mismas deudas y no va a responder por mis hijos, y en el momento en el que él quiera va a decir, «ella se desapareció y no me ha mandado nada y los niños entonces me corresponden a mí».

Cuando logran reunirse en España, contrario a lo que podría esperarse, Diana, la esposa, afirma que se han presentado cambios importantes entre ella y su esposo. Ahora sí toman las decisiones conjuntamente, pues en Colombia era ella la que decidía sin consultarle a él, precisamente porque era ella la que aportaba la mayor parte del dinero para los gastos del hogar. De esta forma, los ingresos de ambos se tienen en cuenta para tomar las decisiones sobre qué se envía a Colombia y a quién, independientemente que en los dos últimos años Humberto haya estado ganando un salario más alto que el de su esposa, llegando en algunos períodos a duplicar el salario de ella. Así, después de cubrir el mantenimiento de los gastos de la casa de Cali, donde viven los niños con la madre de Diana y descontando una pequeña suma que Humberto envía con regularidad a su madre, entre los dos deciden cuánto dinero extra se manda cuando algún familiar de él o de ella solicita una ayuda.

En el hogar de los Caicedo, lo fundamental es que la pareja tiene como regla básica que cada uno maneja su propio sueldo y decide cómo gastarlo, teniendo en cuenta un acuerdo de asignación de los gastos de mantenimiento del hogar. Ella es la encargada de los gastos de la alimentación; él, de pagar el alquiler y los servicios; otros gastos, como el de vestuario sobre todo, los asume ella, aunque a veces le pide colaboración a él. Esta forma de funcionamiento es muy parecida a la de la pareja anterior, con la diferencia que los salarios de Liliana siempre han sido muy bajos. No obstante, como ella ha tenido una mayor estabilidad en el empleo en algunas temporadas, también ha sido la principal aportante al presupuesto familiar. Según ha transcurrido la historia de esta pareja, las decisiones sobre los gastos han dependido mucho de quien esté 
en ese momento percibiendo mayores ingresos, porque en esta pareja cada uno decide sobre su salario, aspecto que no ha cambiado con el proceso migratorio. Así, en los primeros seis meses después de la llegada de Iván a España, él enviaba remesas a su esposa para pagar el billete aéreo y cubrir la deuda de la casa.

Después del accidente de Iván, es Liliana quien asume la responsabilidad por el mantenimiento de los hijos ${ }^{21}$ y el pago de las deudas en Colombia, así como por los gastos del sostenimiento de Iván en España. Su capacidad económica para asumir los gastos, es la que le permite tomar decisiones importantes, aún sin el acuerdo de su esposo, como es el ejemplo del viaje de sus dos hijos a España. A Liliana, sus hijos le decían por teléfono que ya estaban empezando a tener problemas de convivencia en la casa de su suegra en Colombia, por lo que decidió empezar a hacer gestiones para llevarlos a España. Además, un tiempo después, se dio cuenta de que pedirían el visado a los colombianos para entrar a España, lo cual dificultaría más la entrada. Iván no estaba de acuerdo con esta decisión, porque no le agradaba la forma en que estaban siendo educados los niños en España ${ }^{22}$.

Claro, él no estaba de acuerdo, pues casualmente que por la educación que había aquí, por todo lo que veíamos aquí, pues los niños cómo eran, cómo se portaban aquí. Pero, mi pensamiento era que mis hijos tenían que estar conmigo, con sus papás y que nadie era responsable de ellos sino yo y él.

A pesar de este desacuerdo, Liliana siguió con sus planes y, antes de que se exigiera el visado de entrada a España para los colombianos, consiguió que sus dos hijos se reunieran con ellos. De esta forma, llegaron un año después de su llegada, algo inusual incluso en ese período, cuando se pasaban más fácilmente los controles de la frontera, pues la mayoría de familias tardaban como mínimo dos años para llevar a los niños a España. Este ejemplo muestra la autonomía que tiene la mujer respecto a decisiones tan importantes como la reunificación familiar.

La fase posterior de este hogar, después de la llegada de los hijos, y cuando Iván consigue un empleo más estable y mejor remunerado que el de su esposa, permite un reequilibrio en la economía del hogar. En esta fase, Iván logra terminar de pagar la hipoteca que pesaba sobre su vivienda y empezar a enviar dinero con regularidad a su madre, que depende enteramente del dinero que

21. En este caso, debido a que los niños están en la casa de la abuela paterna, los recursos que envió Liliana a Colombia mientras sus hijos vivían en este país, se gestionaron a través de una de las tías paternas.

22. Muchos padres y madres colombianos se sorprenden por la excesiva permisividad de los padres españoles y tienen la imagen de que los niños son irrespetuosos con sus padres, que tienen todo lo que desean y no hacen caso a nadie. Este punto de vista es muy común entre los padres colombianos, que expresan valores más tradicionales respecto al comportamiento de los niños frente a los mayores, sobre todo reforzados porque, en los sectores populares de donde proceden estos migrantes en Colombia, la autoridad paterna es autoritaria, reforzada por una religiosidad y un ámbito doméstico patriarcales. 
le dan sus hijos. Esporádicamente, Iván envía remesas a otro hijo mayor de edad, resultado de una relación anterior ${ }^{23}$. Sobre el dinero que envía a su familia, el arreglo con su esposa es que ella no interfiere mientras él cumpla los compromisos con el hogar en España. Por su parte, Liliana rara vez envía remesas a Colombia después de la llegada de sus hijos, en parte porque sus padres ya murieron y sus hermanas y hermanos no tienen demasiadas afugias económicas, y en parte porque sus ingresos son menores, pero, al igual que Iván, tiene total autonomía en el manejo de sus ingresos, siempre que cubra su parte de gastos en el hogar. De acuerdo con lo que relatan Liliana e Iván, la forma de funcionamiento de ellos no ha cambiado, ni en la distribución de responsabilidades económicas, ni en la forma como toman las decisiones.

Es importante resaltar, en el análisis de las dos parejas anteriores, que se trata de hogares de generaciones distintas y en dos momentos diferentes. En un caso, como se pudo ver en la primera parte, se trata de una pareja más joven: ella con 38 y él con 44 años. Los dos tienen un hijo de 13 y otro de 16 años. La segunda pareja tiene las edades respectivas de 51 y 50 años, con hijos de 12 y 21 años. Esta diferencia generacional puede tener incidencia en los cambios de la primera en comparación con una menor apertura frente a las costumbres de la sociedad española de la segunda. No obstante, como se verá más adelante, en el terreno de los afectos, la segunda pareja también ha sufrido cambios significativos.

Los miembros del hogar Fuentes, en el momento de la entrevista, cuando aún no han logrado la reagrupación familiar en España, siguen funcionando como lo venían haciendo, cada uno manejando sus ingresos, pero teniendo un dinero en conjunto con el que realizan los proyectos en común, ahorrando una suma para el viaje de la esposa y el hijo. Esta pareja, mucho más joven que las anteriores, ella con 34 años, él con 40, tiene un hijo de 10 años. César, quien es el miembro que se encuentra en España, es criticado por su familia materna, porque, aparte de su esposa, no envía remesas ni a su madre, una mujer viuda que vive de una escasa pensión, ni a ninguna de sus hermanas. Aparentemente, podría obedecer a un esquema de valores menos tradicional, con mayor tendencia a consolidar su propio núcleo familiar. Sin embargo, puede corresponder más al contexto en el que se encuentra el hogar, mientras se logra la reunificación familiar.

Las dos familias encabezadas por mujeres presentan dos situaciones que difieren en relación con el ciclo de vida familiar. Mientras, en el caso de Martha, sus hijos ya están en la adolescencia y una es mayor de edad, las hijas de Diana están apenas en la infancia. En el primer caso, después que los hijos de Martha en Colombia dejaron de vivir en la casa de un tío paterno y regresaron al barrio donde antes residían, la gestión de los recursos que ella enviaba se convirtió en un problema. Aparece la rivalidad entre las dos hijas mayores, al confiar

23. Este hijo tiene conformada su propia familia con hijos menores, pero no tiene empleo estable y constantemente llama a España para pedirle a su padre que le envíe dinero. 
Martha la responsabilidad del manejo de los recursos a la menor de ellas, por considerar que era más responsable. Esto la obligó a llevarse a su hija mayor a España. Ahora, el dinero lo sigue enviando a la misma hija, que se encarga de distribuirlo entre sus gastos, los de su sobrina, hija de la hermana que está en España, y los del hermano menor.

En el hogar de Olga, ha sido una de sus hermanas la persona que se ha encargado siempre de recibir y administrar las remesas que envía, ya que su suegra, por tener una avanzada edad, padece dificultades para ir a reclamar el dinero que ella despacha con regularidad. A través de su hermana, envía el dinero para los gastos de sus niñas y el pago de la empleada doméstica que colabora en su cuidado, para algunos gastos de su suegra y la compra de una casa, más los arreglos que ha requerido, y, finalmente, para imprevistos de alguna de sus hermanas, que a veces le piden ayuda. En este sentido, Diana ahora ha cobrado mayor importancia en su familia. Ella es vista como un modelo de éxito, pues de ser una persona que apenas sobrevivía con sus ingresos como secretaria, en pocos años logró adquirir una vivienda ubicada en un barrio residencial de clase media baja en la ciudad de Cartago.

En el análisis del tema de las relaciones de género en la gestión de los recursos económicos, hemos podido ver dos factores que tienen incidencia en los ejemplos expuestos. De un lado, el componente generacional es un elemento importante en la visión que tienen las mujeres y los hombres respecto a los roles que deben cumplir, tanto en el ámbito del trabajo remunerado como del trabajo doméstico. Hemos visto que en los hogares en los que las parejas tienen mayor edad, como era de esperar, hay una visión más tradicional respecto al papel del hombre como proveedor principal, un hecho que, antes de la migración, estaba generando dificultades en la pareja y que se resuelve en el país receptor. Sin embargo, como pudimos ver, esto no significa que las mujeres pierdan poder respecto a la toma de decisiones a partir del proceso migratorio, todo lo contrario, éste ha generado nuevas situaciones en las que las mujeres han tomado parte activa en las decisiones y no se muestran dispuestas a renunciar a hacerlo, a pesar de haber reducido su tiempo y sus ingresos mediante el trabajo remunerado. Ahora bien, en los hogares con parejas más jóvenes, en un caso vimos que había una visión menos rígida del papel del hombre como proveedor principal de los recursos económicos, en la que se reconoce que la mujer, por sus capacidades, puede, en determinados momentos, convertirse en la proveedora principal del hogar, sin que esto genere conflictos en el interior de la pareja. En el otro, la pareja ha logrado conciliar una repartición más equitativa de los roles domésticos. El segundo factor que desempeña un papel importante en las relaciones de género, es la capacidad de las mujeres para generar sus propios ingresos, y el hecho que en algunas etapas éstos hayan superado al de los esposos, ha permitido a las mujeres tener una voz firme en la toma de decisiones. Pero, como veremos en el siguiente apartado, el factor económico no es necesariamente el único determinante, ya que, en la negociación sobre el uso de los recursos económicos, está imbricado el campo afectivo. 


\section{La recomposición de los afectos y la sexualidad}

En este apartado, analizaremos, en primer lugar, las parejas que han logrado reagruparse en España, que corresponden a la familia Bejarano y a la familia Caicedo. En estos dos casos, el proceso migratorio y la situación en el nuevo contexto han permitido la renegociación de la relación de pareja y la recomposición de los afectos que venían en crisis de tiempo atrás.

Veamos cómo ha sido el proceso de la pareja del hogar de los Bejarano. En el período previo a la emigración, la pareja sufría un proceso de desgaste cuando vivía en Colombia y que Diana atribuye a la desidia de Humberto para responder por la responsabilidad económica que le correspondía en el hogar, sumado al consumo de alcohol y a las infidelidades. Ésta es la respuesta de Diana a las razones por las que ella aportaba la mayor parte del presupuesto al mantenimiento del hogar en Colombia:

Él ganaba menos y no le interesaba cubrir los gastos de la casa, porque él sabía que yo iba a responder y estaba en otro cuento, él era el trago, las mujeres.

Diana estaba sobre todo cansada de asumir siempre la responsabilidad económica, incluso cuando ella quedó sin trabajo y le expuso a su marido la necesidad de que uno de los dos buscara otras posibilidades fuera del país. Sin embargo, él se niega a viajar. Ella, en su relato sobre las motivaciones que tuvo para emigrar, además de la situación económica, enuncia otra causa, el proceso de desgaste que atravesaba su relación de pareja:

Pero yo me fui más decepcionada de mi matrimonio que otra cosa. Mejor dicho, yo me fui pensando en que las cosas estaban más rotas que arregladas.

No obstante, cuando Diana emprende su viaje, decide no plantear una ruptura, porque tiene miedo que su esposo la acuse de abandono del hogar y pierda el derecho sobre sus hijos. Un tiempo después, cuando ella está en España, de nuevo le propone que viaje él y se quede trabajando para que ella pueda volver con los niños, pero él también se niega a esta opción. Por eso, cuando ella empieza a percibir que su marido en Colombia ya no hace frente a la responsabilidad mínima que habían acordado, le habla de ruptura:

Claro, entonces, hasta que yo le dije a él que no, que esa situación así no, que yo consideraba que yo me había casado para tener un hogar y que todo era por partes iguales, que así como yo me estaba sacrificando por mis hijos tan lejos, que él también tenía que poner de su parte, que no, que yo veía que las cosas no iban a más y que él tampoco, y que entonces no más.

Después de plantearle a su marido la ruptura de la pareja, Diana abiertamente le formula a él su deseo de no regresar, pero le pide que le permita llevarse a los niños a España. Su marido le niega la posibilidad de llevarlos a España con ella si se separan y empieza a chantajearla emocionalmente con los niños: 
Entonces, él, como en represalia, se fue a vivir solo ${ }^{24}$ con los niños, y empezó a decirles cosas a los niños, y los niños de hecho cambiaron conmigo. Yo llamaba y el niño no se ponía al teléfono, no hablaba conmigo, por todo lo que él les dijo. El les dijo que sí, que seguro yo allá tenía una relación, que seguro yo ya me había conseguido un amante, les llenó la cabeza de cosas. Que yo no los quería, que yo no iba a volver.

Ella, a pesar del dolor que le causaba la situación ${ }^{25}$, de todos modos estaba dispuesta a dejar de ver a sus hijos durante un tiempo largo. Al ver que ella no se amilana, Humberto empieza a dar su brazo a torcer y le plantea a su esposa el deseo de ir a España, lo cual, como se describió antes, consiguió Diana con un precontrato de trabajo. Sin embargo, ella le deja claro que, si él viaja a España, no va a garantizar la continuidad de la relación, que eso dependerá de si, en el reencuentro, los dos logran entenderse de nuevo.

En el caso de esta mujer migrante, es necesario observar los efectos del proceso de individuación que genera la experiencia migratoria transnacional. Por primera vez en muchos años, Diana había tenido la oportunidad de estar sola y de reflexionar sobre sí misma y sobre la vida que llevaba, al punto de llegar incluso a dudar de sus propios sentimientos:

Pensaba también en la relación como pareja, tantos años separados, yo decía: "¿Será que lo quiero, será que no lo quiero? ¿Será que todo lo que viví antes era como no sé..., como algo que no sentía en verdad?».

La posibilidad de «mirarse a si misma» es lo que le permitirá luego exigirle con claridad a su esposo cuáles son sus verdaderos intereses y demandarlo a romper con lo que había sido su comportamiento en el hogar durante la convivencia de la pareja en Colombia. Humberto viaja a pesar de las reticencias de su esposa y, como describe Diana, a su llegada, ella «lo sentó» durante todo un día y le expuso sus condiciones, qué era lo que ella quería para su vida y cómo consideraba que debía funcionar su hogar:

Y así, cuando él llegó, yo lo senté y hablé con él, todo un día. Él llegó por la mañana y todo el día hablamos, y yo le dije mis puntos de vista son estos, estos y estos, y yo pienso que mi hogar debe de ser así y que la relación así, y que el compromiso, y yo le dije, el hombre de la casa es usted, no soy yo, yo no tengo porque responder por las cosas.

Humberto admitió los reclamos de su esposa y aceptó las nuevas reglas del juego. Para Diana, esto no era suficiente y seguía desconfiando del compromiso de su marido, pero, con el tiempo, pudo darse cuenta que él realmente estaba dispuesto a recuperar su relación y que se había tomado en serio los nuevos compromisos adquiridos con ella. Humberto cambió total-

24. Inicialmente, Humberto vivía con la madre de Diana y los niños.

25. De acuerdo con su relato, en ese período, Diana intentó suicidarse. 
mente respecto a lo que era su comportamiento en los anteriores años de matrimonio:

Él me dijo, yo sé que usted tiene la razón en todo, y ya me dijo que él había dejado aquí unas deudas y que él quería pagarlas. De hecho, en los seis primeros meses las pagamos, pero ya fue diferente, ya él recibía el sueldo, y aún sigue igual, el recibe el sueldo y yo soy la que lo distribuyo...Uy, a él le cambió el chip totalmente la vida en España ${ }^{26}$. Yo vivo aterrada a veces, y es organizado, él antes no se preocupaba si debíamos mil, dos mil o tres mil. No, él ahora dice: «Diana, cero deudas, Diana, tenemos que cumplir estas metas».

Pero la relación no cambió sólo en el sentido de asunción de responsabilidades en el hogar, sino en el ámbito de lo afectivo. Para esta pareja, el nuevo contexto ha permitido un acercamiento diferente, otras prácticas con otras formas de expresión del afecto en la cotidianidad que antes no tenían, como relata Diana en la siguiente cita:

Cambió mucho, porque nosotros nos habíamos alejado demasiado y allá pues nos volvimos a unir, él se volvió más cariñoso, más pendiente de mí, igual yo de él, ya nos tratamos con palabras cariñosas, que antes era Diana y Humberto, ya no más. Sí, habíamos enfriado mucho la relación. También ya ahora hay otra cosa y es lo que antes no hacíamos aquí en Colombia y es que salimos juntos a todo, que hoy vamos a ir al cine o que vamos a ir a comer, que vamos a ir a hacer la compra, todo lo hacemos juntos. Ahora tenemos una vida en pareja.

En el hogar de los Caicedo, podemos encontrar otro ejemplo de recomposición de la pareja a partir del proceso migratorio. A diferencia de los Bejarano, Iván, que ya había emprendido proyectos migratorios anteriormente, es el que viaja inicialmente a España. En este caso, también está la crisis de pareja sumada a la situación de precariedad laboral de Iván. Liliana le había dado un ultimátum a su marido:

Yo le dije que se fuera, era mujeriego, me pasaba las mujeres por delante de la casa, tomaba mucho, tenía una moto y se la pasaba montando viejas [mujeres] a la moto, no trabajaba.

Liliana afirma que todavía no sabe cómo ella ha seguido con él durante tantos años, teniendo en cuenta que él se portaba tan mal en Colombia. Esta crisis matrimonial se resolvió cuando ella viajó a España por el accidente de su marido. Ella estuvo dispuesta a dejarlo todo por ir a hacerse cargo de él, dejó un trabajo estable y a sus hijos. Tuvo que trabajar interna durante un año mientras Iván se recuperaba. Esta demostración del compromiso con su espo-

26. La entrevista fue realizada en Cali cuando ella vino de vacaciones con su marido. Él no figura en las citas, porque se incomodaba mucho con el uso de la grabadora. 
so, así como el hecho que Iván, después de recuperarse, pudo conseguir un empleo y la pudo relevar a ella de la mayor parte de la carga económica del hogar, fueron factores importantes en la recomposición de esta pareja:

Pues ahora yo siento que estamos un poquito mejor, porque ya se han solucionado muchas cosas y al final ya tenemos a los hijos aquí, y ya trabaja él, ya trabajo yo, ya logramos pagar la casa en Colombia, que era lo que más nos preocupaba, eso ya se hizo, y ahí poquito a poco vamos saliendo de la mala racha.

Si bien Liliana no puso explícitamente nuevas condiciones a su esposo, de alguna manera, el nuevo contexto ha implicado una nueva vida para ellos como pareja, que simbólicamente se ratificó con el rito del matrimonio por la Iglesia ${ }^{27}$. Ella considera que su marido ha cambiado, por lo que se ha dado una considerable mejoría en las relaciones entre ellos:

Sí, ha cambiado muchísimo, referente a que mantenía en la calle sinvergüenciando ${ }^{28}$, ahora aquí no, porque, cuando toma, toma aquí en la casa y ya. Estamos como más juntos, en Colombia era él por su lado y yo por el mío, en cambio aquí, pues no. Para saber muchas parejas que aquí se han desbaratado, pero en cambio, nosotros ha sido como al contrario.

Iván también reconoce que ha cambiado, pero lo atribuye a que es muy difícil ganarse el dinero para malgastarlo luego y que carece de un grupo de amigos con quienes salir. Ella más bien lo atribuye a que en España su marido no tiene éxito con las mujeres:

Pues él dice que porque aquí no se puede gastar el dinero así por así y que, pues, les toca trabajar muy duro para ganarse el dinero, para írselo a gastar por ahí, y otra cosa que él no dice es que las españolas no les paran bolas ${ }^{29}$ a los colombianos.

Esto último posiblemente tiene que ver con que en España, por las relaciones de género prevalecientes, los hombres colombianos no tienen el mismo estatus que en el país de origen y por ello se encuentran desvalorizados en el mercado matrimonial o de parejas, pues las mujeres españolas y la mayoría de extranjeras no están dispuestas a relacionarse con un hombre de un estatus inferior al de ellas. Por otro lado, en la situación opuesta, es menos condenable que un hombre entable relación con una mujer de un estatus inferior, a lo que se suma el hecho que en determinadas regiones ${ }^{30}$ o en grupos de edad específicos hay un fuerte desequilibrio demográfico entre hombres y mujeres.

27. Liliana e Iván llevaban más de quince años como pareja de hecho.

28. Expresión que alude a un comportamiento infiel, casi permanente.

29. Expresión que significa que no los miran, no les prestan ninguna atención.

30. Especialmente en las zonas menos urbanizadas. 
Por otra parte, hay un cierto imaginario sobre las mujeres latinoamericanas que, por un lado, son consideradas más sumisas que las mujeres españolas y, por otro, se cree que sexualmente son más activas. Todos estos ingredientes llevan a que las mujeres latinoamericanas, y en general las extranjeras, tengan más oportunidades de encontrar compañero afectivo o sexual que los hombres extranjeros, lo cual, en las relaciones de pareja del mismo país, otorga cierta ventaja a las mujeres cuando los afectos se renegocian y se recomponen. Por ejemplo, Liliana, a pesar de estar bordeando los cincuenta años, ya ha sido abordada por un vecino español, dueño de un bar en su barrio, algo que hasta ahora no le ha sucedido a Iván con ninguna mujer española.

En relación con las mujeres solas, queremos resaltar el caso de Olga, pues, a pesar de ser una mujer víctima de violencia doméstica, constreñida en su comportamiento por sus creencias religiosas de una secta cristiana, la convivencia en España con otras mujeres colombianas de mayor nivel educativo y otra forma de pensar distinta a la de ella, en asuntos como el ejercicio de la sexualidad y la actitud que deberían tomar las mujeres en sus propias decisiones, así como lo que ella ha podido observar en la sociedad española, distinta a la suya, le permitieron gradualmente pensar en la posibilidad de rehacer su vida y dejar de sentir culpabilidad ante la posibilidad de llevar relaciones sexuales por fuera del matrimonio que dejó en Colombia.

La distancia propicia varias cosas. Por un lado, el hecho de escapar al control social familiar y de la sociedad de origen, permite a las mujeres tener conductas que nadie de su entorno familiar va a tener la oportunidad de censurar. Por otro lado, la posibilidad de conocer a hombres y a mujeres de otros contextos sociales y culturales, con prácticas distintas respecto al amor y a la pareja. En este caso, un tiempo después de llegar a España, Olga empieza a salir con amigos de España y de la República Dominicana en plan de búsqueda de pareja, algo que no se hubiera atrevido a hacer en su pequeña ciudad, donde era muy fácil ser reconocido por familiares y amigos, pero no sólo por la censura de éstos, sino también por la de su ex marido, que, a pesar de tener ya otra pareja, vigilaba su conducta. Las nuevas experiencias, en un contexto completamente diferente al de la sociedad de origen, permiten reconocer que las otras personas no se comportan como se piensa espontáneamente y que tampoco necesariamente se ajustan a las creencias que traen, según el sentido común, sin que por lo mismo se piense que buscan aprovecharse de la otra persona. Es decir, que la vivencia de la sexualidad con placer no significa compromiso ni utilización de la otra persona:

Entonces, pues yo pensaba que hasta que no me casara por mi iglesia, yo no iba a tener relaciones sexuales con otro hombre, porque con el papá de mis hijas no estaba casada. Pero, con el tiempo, me di cuenta que ningún hombre que no fuera de mi iglesia se aguantaba eso. En general, los hombres lo primero que quieren es tener relaciones con una, pero incluso los que son menos acelerados se cansan de que una les diga que no y mucho menos van a esperar a un matrimonio, además porque yo ya tengo hijos, eso no lo entienden. 
Esa distancia, no sólo física sino también mental, que se toma respecto a la sociedad de origen, da a las personas que emigran la capacidad de reflexionar sobre el pasado, el presente y la vida que se desea, la posibilidad de un cambio en el campo de los afectos y los deseos. Así, como la vida llega a dar un giro en la vida material, hay también cambios en la forma personal de asumirla. Se empieza a tener en cuenta el sentido práctico de lo que se quiere y a dejar a un lado las consideraciones morales de tipo cultural. Ese giro respecto a lo que valora, al peso que da a cada cosa, es lo que va a permitir a Olga la posibilidad de iniciar una relación de pareja, con unos parámetros muy distintos a los que tuvo con su primer compañero:

También comencé a averiguar cómo traer a mis hijas a España y me di cuenta que yo sola, con el sueldo que podía ganar limpiando escaleras o haciendo limpieza, yo sola nunca iba a poder tener condiciones para traerlas. Por eso fui cambiando de mentalidad, yo no podía ponerme a esperar a ver si en mi iglesia encontraba a alguien, porque mis hijas me hacen mucha falta. Empecé a ser un poquito más abierta... Por eso, cuando conocí a Alberto ${ }^{31}$ y que me pareció buena persona, ya no me puse con tantas pendejadas [tonterías] y, aunque él no pertenece a mi iglesia, decidí seguir para adelante con él. Alberto es muy distinto al papá de mis hijas, es un hombre respetuoso, tranquilo y responsable, organizado con el dinero. Ahora que ya puedo traer a mis hijas, porque ya tengo papeles, nos hemos puesto de acuerdo para comprar un piso.

Otro ejemplo que nos puede servir para mostrar procesos de individuación y subjetivación y de autonomización de la mujer, a partir del fenómeno de la migración, se encontró en una migrante de Candelaria, municipio del área metropolitana de Cali. Se trata de una mujer mestiza de 26 años, trabajadora sexual en España, quien, desde que entró en la prostitución, puso al tanto a su familia y, frente a sus cuestionamientos morales, argumentaba que ellos (su madre y su hermano) dependían económicamente de ella y que la remuneración que recibía le permitía sostenerlos desde España. Pero, en el caso de esta mujer, no se trataba sólo de un argumento para justificarse ante la familia, pues al ser interrogada sobre su propios sentimientos respecto a la vida que llevaba en España como trabajadora sexual, aparte del beneficio económico de la actividad que ella desarrollaba, destacaba el hecho que en ese trabajo conocía sitios hermosos, gente totalmente distinta. Además, respecto al ejercicio de su sexualidad, ella consideraba que, aunque estuviera supeditado a los requerimientos del cliente, era realmente agradable para ella, porque le permitía explorar formas de sexualidad totalmente extravagantes, diferentes y divertidas. En este caso, como en el anterior, este nuevo posicionamiento ante sí mismas, ha posibilitado a estas dos mujeres tener una nueva actitud ante el ejercicio de la sexualidad.

31. Alberto es un hombre peruano de 40 años. 
En los dos primeros casos analizados, se pudo observar, en primer lugar, las estrategias utilizadas por las mujeres para maniobrar en una relación insatisfactoria pero que no desean romper abruptamente por la presencia de los hijos, insatisfacción que no sólo tiene que ver con lo económico, sino también con lo afectivo, la distancia que se ha ido marcando en las parejas con el paso del tiempo, las dificultades y la rutina. La migración, sea del hombre o de la mujer, va a permitir romper con ese estado de cosas, tomar distancia y reflexionar, va a enseñar otras maneras de relacionarse, a mostrar otras posibilidades a las mujeres revalorizadas en un mercado matrimonial que tiene distintos parámetros frente al contexto local de origen. Por lo tanto, no se trata sólo de una renegociación de la relación, sino de resituarse cada uno y ver al otro desde una posición distinta, de esta forma permitirse a sí mismo nuevas maneras de hablar, de sentir, de actuar en el espacio afectivo ${ }^{32}$. También, en el caso de las mujeres que emprenden el proyecto migratorio en solitario, podemos ver esa especie de ruptura que propicia la migración, el mirarse a sí misma la mujer, actuar y sentir de forma distinta. En este sentido, las prácticas que desarrollan las personas para hacer frente a una situación laboral precaria en el país de origen, las obliga a aceptar el riesgo, a aprovechar las oportunidades que se presentan en cada momento, incluyendo un correlato en el terreno de lo subjetivo, lo íntimo, que modifican paulatinamente las valoraciones morales que, por lo mismo, pueden ser cambiantes, para hacer posibles los deseos propios que ahora se reconocen más claramente.

\section{Conclusiones}

Los emigrantes colombianos proceden de hogares de clases populares (la región Pacífica, y en particular el Valle del Cauca, tienen la mayor participación en la ola migratoria hacia España, sobre todo desde finales de la década de 1990), sin ser los sectores más pobres. Se trata de hogares que, en una buena parte, los hombres han enfrentado problemas para asumir el rol que tradicionalmente se les ha atribuido como proveedores económicos en una sociedad como la colombiana. La actitud asumida por ellos, en la mayoría de los casos, ha sido de evasión o de huida, lo que ha llevado a que las mujeres asuman este papel como principales generadoras del ingreso o incluso asumiendo el papel como cabezas de familia, tanto del hogar en España como el de los miembros que han quedado en Colombia. Este antecedente, en la dinámica de las parejas, ha sido uno de los factores que ha desencadenado la migración a escala micro de la familia, como parte de una crisis no sólo en el plano económico, sino también en el ámbito afectivo. En ciertas condiciones, para las parejas que han logrado reunirse en España en los ejemplos analizados, pudimos observar que

32. Vale la pena aclarar que, si bien en la investigación que se adelanta, se encontraron casos en los que las parejas en España se fortalecieron, existen muchos casos, tal como lo manifestó una de las entrevistadas, en los que no sólo las relaciones afectivas y de pareja, sino también las familiares, se rompieron. 
los hombres tienen alternativas de encontrar empleos mejor remunerados que los de sus esposas, una vez se han regularizado y han logrado insertarse mejor en el mercado laboral. Este elemento les ha permitido retomar su papel en el hogar en España como proveedores económicos principales. El diferencial salarial significativo a favor de los hombres ha incidido en una reducción en la jornada de trabajo de las mujeres que tienen su pareja en España, contrariamente a lo que pasa con las mujeres que emigran a España como cabezas de familia en los dos países, ya que deben intensificar su jornada de trabajo para poder mantenerse y tener dinero suficiente para sostener al resto de miembros del hogar en el país de origen. Esto último dificulta enormemente a las mujeres que están solas, y aplaza la posibilidad de realizar la reagrupación familiar con los hijos.

De todos modos, en las parejas, detrás de la aparente o «real» asunción de responsabilidades por parte de los hombres, se ha dado todo un proceso de renegociación de las reglas del juego en las que las mujeres, si bien han podido perder terreno en la percepción de los ingresos, lo han ganado en la toma de decisiones sobre la utilización de los recursos generados por ambos miembros de la pareja. Es importante resaltar que la capacidad de las mujeres de generar sus propios ingresos ha incidido en su incidencia en la gestión de los recursos económicos y en otras decisiones frente a la organización del cuidado de las personas dependientes del hogar en Colombia. En general, se puede afirmar, desde el punto de vista de las relaciones de género, que las mujeres que emigran ganan prestigio dentro del hogar transnacional por su capacidad para aportar nuevos o mayores ingresos respecto al país de origen.

Es bueno advertir que, el reparto de las tareas domésticas en España, aún continúa siendo asumido con un mayor tiempo de carga laboral por parte de las mujeres colombianas en la mayoría de los casos, y, en el país de origen, entre las mujeres de la parentela materna o paterna, a pesar de haberse producido algunos cambios en la división del trabajo doméstico, sobre todo en España. Observamos que hay diferencias generacionales en la actitud respecto a la división del trabajo, tanto asalariado como doméstico, con posturas menos ajustadas a los roles tradicionales entre las parejas más jóvenes.

También, se pudo observar, a partir de los resultados, que la renegociación de la que hablábamos atrás en el terreno de los recursos económicos y de la organización doméstica de los hogares transnacionales está imbricada con las posiciones que se toman en el terreno de lo afectivo, de lo íntimo, de las creencias y los sentimientos, al igual que en la vivencia de la sexualidad. La transformación de las relaciones en el interior del hogar a que da lugar el proceso migratorio permite a los sujetos situarse en un lugar distinto frente al otro. Gracias a ello, se generan nuevas actitudes y, ante todo, prácticas en varios aspectos de la vida cotidiana. Por esta razón, emergen nuevas identidades y subjetividades, procesos de autonomización en estos sectores de las clases populares colombianas, en lo que recientemente se conoce como las «identidades flexibles» que se producen en los contextos transnacionales. En tal sentido, de acuerdo con Sassen (2003a: 49), los trabajos más recientes sobre la migración 
internacional comienzan a centrarse en el examen del hogar, ya que esta categoría analítica permite «entender los procesos económicos globales y las nuevas formas de solidaridad transfronteriza, las experiencias de pertenencia y formación de la identidad que representan las nuevas subjetividades, incluyendo las subjetividades feministas». Igualmente, la revalorización de las mujeres versus la devaluación de los hombres en el «mercado matrimonial», dependiendo de la edad y del ciclo de vida de éstos últimos y del contexto de llegada, ha sido un elemento importante en la recomposición de los afectos, en las nuevas actitudes frente a la pareja, los hijos y la sexualidad, con efectos en el comportamiento de hombres y mujeres.

Por lo anterior, los hallazgos de este artículo apuntan en la misma dirección anotada por Sassen (op. cit.: 71): «efectivamente, asistimos al nacimiento de una nueva fase en la producción de conocimiento sobre las mujeres y la economía global, esta vez respecto a los procesos que subyacen a las transformaciones en las relaciones de género, las subjetividades de las mujeres y sus nociones de pertenencia. Todo esto comprende corrientes intelectuales muy distintas. Entre las más ricas y prometedoras, se encuentran las nuevas investigaciones feministas sobre las mujeres inmigrantes, que se focalizan, por ejemplo, en cómo las migraciones internacionales alteran las conductas de género y cómo la formación de hogares transnacionales puede fortalecer a las mujeres».

\section{Bibliografía}

Aparicio G., Rosa; Giménez R., Carlos (2003). Migración Colombiana en España. Universidad de Comillas, Universidad Autónoma de Madrid, OIM y Naciones Unidas, Ginebra, 220 p.

BACA, Jorge y otros (2005). Estudio sobre Migración Internacional y Remesas en Colombia. Metodología Encuesta sobre Emigrantes Internacionales y Remesas en el Área Metropolitana Centro Occidente. AMCO Metodología Encuesta a Beneficiarios de Remesas de Trabajadores en Instituciones Cambiarias de Colombia. Departamento Administrativo Nacional de Estadística (DANE). Organización Internacional para las Migraciones (OIM). ISBN 958-33-7538-I, Bogotá, 140 p.

DANE (Departamento NaCiOnAl de Estadística). Resultados preliminares Censo 2005. <www.dane.gov.co/files/censo2005.>

GARAY, Luis Jorge; RODRÍGUEZ, Adriana (2005a). Estudio sobre Migración Internacional y Remesas en Colombia. La Migración Internacional: Una Síntesis de Aproximaciones Teóricas Alternativas; La Emigración Internacional en Colombia: Una Visión Panorámica a partir de la Recepción de Remesas. Ministerio de Relaciones Exteriores de Colombia. Organización Internacional para las Migraciones (OIM). ISBN 95833-7539-X.

- (2005b). Estudio sobre Migración Internacional y Remesas en Colombia. La Emigración Internacional en el Area Metropolitana Centro Occidente Colombia. Caracterización Socioeconómica de la Población Emigrante y Evaluación del Impacto de las Remesas Internacionales. República de Colombia, Ministerio de Relaciones Exteriores; Presidente de la República, Álvaro Uribe Vélez; Ministra de Relaciones Exteriores, Carolina Barco Isakson; Programa Colombia Nos Une, Ministerio de Relaciones Exteriores, 80 p. 
RAMírEZ, Carlota y otros (2005). Cruzando fronteras: Remesas, género y desarrollo. Marta Ramírez, Jeannie Ash de Pou, editoras. Instraw, NU, Santo Domingo. República Dominicana, $70 \mathrm{p}$.

SASSEN, Saskia (2003a). Contrageografías de la globalización. Género y ciudadanía en los circuitos transfronterizos. Madrid. Traficantes de Sueños.

Urrea Giraldo, Fernando; OrTiz, Carlos (1999). «Patrones sociodemográficos, pobreza y mercado laboral en Cali (documento de trabajo para el Banco Mundial)». Cidse. Banco Mundial. Universidad del Valle, 70 páginas. Sin publicar.

\section{Otras fuentes:}

Cinco entrevistas realizadas en Cali, Cartago y Palmira por la antropóloga Jenny Posso Quiceno.

Dos entrevistas llevadas a cabo en Candelaria por el estudiante de sociología Alexander Castaño López. 